\title{
Paternal Occupational Exposure to Endocrine-Disrupting Chemicals as a Risk Factor for Leukaemia in Children: A Case-Control Study from the North of England
}

\author{
Mark S. Pearce, ${ }^{1}$ Lucy R. Baxter, ${ }^{1}$ and Louise Parker ${ }^{2}$ \\ ${ }^{1}$ Institute of Health \& Society, Newcastle University, Sir James Spence Institute, Royal Victoria Infirmary, \\ Newcastle upon Tyne NE1 4LP, UK \\ ${ }^{2}$ Departments of Medicine and Paediatrics, Dalhousie University, Halifax, NS, Canada \\ Correspondence should be addressed to Mark S. Pearce; mark.pearce@ncl.ac.uk
}

Received 23 April 2014; Accepted 8 July 2014; Published 16 July 2014

Academic Editor: Peter N. Lee

Copyright (C) 2014 Mark S. Pearce et al. This is an open access article distributed under the Creative Commons Attribution License, which permits unrestricted use, distribution, and reproduction in any medium, provided the original work is properly cited.

\begin{abstract}
Occupations with exposures to a variety of chemicals, including those thought to be potential endocrine disruptors, have been associated with an increased risk of leukaemia in offspring. We investigated whether an association exists between paternal occupations at birth involving such exposures and risk of leukaemia in offspring. Cases $(n=958)$ were matched, on sex and year of birth, to controls from two independent sources, one other cancers, one cancer-free live births. Paternal occupations at birth were classified, using an occupational exposure matrix, as having "very unlikely," "possible," or "likely" exposure to six groups of potential endocrine-disrupting chemicals. There was a significantly increased risk of acute nonlymphocytic leukaemia (ANLL) for polychlorinated organic compounds (OR 1.95, 95\% CI 1.08-3.54) only in comparison with cancer-free controls, and for phthalates (OR 1.61, 95\% CI 1.00-2.61) only with registry controls. A number of other, including inverse, associations were seen, but limited to one control group only. No associations were seen with likely paternal exposure to heavy metals. The associations identified in this study require further investigation, with better exposure and potential confounding (for example maternal variables) information, to evaluate the likelihood of true associations to assess whether they are real or due to chance.
\end{abstract}

\section{Introduction}

Exposure to a number of chemicals, collectively known as "endocrine disruptors," is suggested to increase the risk of a number of adverse health outcomes through interference with the endocrine system $[1,2]$. Examples of potential endocrine-disrupting chemicals include organochlorine and organophosphorus pesticides, polychlorinated biphenyls (PCBs), dioxins, phthalates, alkylphenols, heavy metals such as cadmium, lead, and mercury, and phytoestrogens [3]. A relatively recent scientific statement by the Endocrine Society stated that endocrine-disrupting chemicals were a "significant concern to public health" [1].

Numerous studies have implied that paternal preconceptional occupational exposures may have a role in the aetiology of childhood cancers [4], though relatively few studies have focused on endocrine disruptors. In those that have, occupations with exposures to a variety of chemicals, including those thought to be potential endocrine disruptors, have been associated with an increased risk of leukaemia in offspring [4-9]. One such group of potential endocrine disruptors is pesticides, herbicides, and fungicides [10]. We have previously reported a negative association between likely paternal occupational exposure to pesticides or herbicides, particularly in males that were no longer significant after adjusting for urban/rural residential status [11]. However, there are a wide range of other chemical exposures thought to have endocrine-disrupting effects, such as polychlorinated organic compounds, phthalates, alkylphenolic and biphenolic compounds, and heavy metals, such as lead. Some of these, 
such as polychlorinated organic compounds [8,9], alkylphenolic compounds $[6,9,12]$, and phthalates [5], have previously been associated with an increased risk of childhood leukaemia in the offspring of exposed men, although other studies have not found these associations [12-18].

We tested the hypothesis that an association exists between paternal occupations at the time of birth with possible exposures to potential endocrine-disrupting chemicals and risk of childhood leukaemia in children $(0-14 \mathrm{yrs}$ at diagnosis) in the North of England using data from the Northern Region Young Persons' Malignant Disease Registry (NRYPMDR) [19] and the Cumbrian births database (CBD) [20].

\section{Material and Methods}

The NRYPMDR is a population-based registry recording young people, aged under 25 years, diagnosed with malignancies and benign central nervous system tumours and resident in the North of England, since 1968 [19, 21]. The registry is located within the Newcastle upon Tyne Hospitals NHS Foundation Trust, which is the regional specialist centre for cancer in children and adolescents. Registration with the registry is not mandatory, but cases are identified from multiple sources; consultants throughout the region notify the registry of any malignancies in children and young adults, and death certificates and hospital admissions are regularly scrutinised. Data are periodically cross-checked with regional and national cancer registries to ensure that the information held is as accurate and complete as possible. Overall ascertainment of cancers is believed to be more than $95 \%$ complete [19]. The study region is a mixture of several heavily populated urban areas and widespread rural communities. It has a population of 3.1 million that are predominantly white (ethnic minorities account for under $2 \%$ ), with approximately 37000 live births per year. The NRYPMDR is exempted (originally under Section 60 of the UK Health and Social Care Act 2001, which has now been superseded by Section 251 of the National Health Service Act 2006) from the need to obtain patient consent for recording and analysis of data.

Cases of leukaemia aged 0-14 years at time of diagnosis and diagnosed between 1968 and 2000 were ascertained from the NRYPMDR. Two independent sets of controls were selected to allow the consistency of findings to be assessed between the control groups used. Firstly, for each particular disease group all other patients from the NRYPMDR with different cancer types, with the same sex and year of birth as each case, were selected as controls. Secondly, 100 controls per case were randomly selected from live births recorded on the $\mathrm{CBD}$ and not diagnosed with cancer during childhood, again matched on sex and year of birth. The CBD, described in detail elsewhere [20], holds birth registration details on all children born to mothers resident in the county of Cumbria, in the North of England, between January 1, 1950, and December 31, 1993. The county of Cumbria contributes to the NRYPMDR and includes approximately $16 \%$ of the population of the north of England. In common with the rest of the study region, Cumbria is a mixture of both urban and rural areas, but is among the most sparsely populated counties in England, with industrial areas concentrated in the south west.

2.1. Paternal Occupation. Paternal occupation, as recorded on birth certificates, which are obtained by the registry whenever possible, was coded according to the 1990 Standard Occupational Classification [22] and hence the paternal occupational social class at the time of the child's birth (I, professional and assumed to be the most advantaged, II and III nonmanual, III manual, and IV and V unskilled and assumed to be the least advantaged) was derived. Those for whom paternal occupation was unavailable from birth certificates, or for which there was no suitable occupational code (including "unemployed" and "student"), were excluded.

A job-exposure matrix previously developed by van Tongeren et al. was used to classify paternal occupations into categories based on the likelihood ("very unlikely," "possibly," and "likely" (probable that at least a proportion of workers had some exposure)) of the occupation involving exposures to potential endocrine disrupting chemicals [10]. As the matrix was published using 1980 occupational classification codes, a conversion table was obtained from the UK Office for National Statistics to allow the matrix to be used. The seven exposure groups in the matrix are as follows: (1) pesticides, (2) polychlorinated organic compounds, (3) phthalates, (4) alkylphenolic compounds, (5) biphenolic compounds, (6) heavy metals, and (7) other hormone disrupting chemicals. As we have previously reported results for the pesticides group [11], only the latter six categories, were considered in this analysis.

2.2. Statistical Analysis. For each exposure category, we investigated whether the risk of childhood leukaemia was increased for paternal employment at birth, likely to result in exposure to an endocrine disrupting chemical, compared to the risk in those occupations within the "very unlikely" group. In performing the analyses, we considered all cases, then males and females separately to explore whether potential associations may differ for male and female offspring. We also report analyses limited to lymphoid and acute nonlymphocytic leukaemia (ANLL). Odds ratios (OR) and corresponding 95\% confidence intervals (95\% CI) were estimated using conditional logistic regression, adjusting for the potential confounding factor of parental occupational social class. Stata, version 12.0, (Stata Corp, College Station, TX) was used for all statistical analyses.

\section{Results}

Table 1 shows the distribution of cases and both sets of control groups in relation to levels of likely exposure to the six groups of potentially endocrine-disrupting chemicals considered in this study. The study included 750 cases of leukaemia (328 female and 422 male), including 624 cases of lymphoid leukaemia (266 female and 358 male) and 101 cases of ANLL ( 49 female and 52 male). Of the 750 leukaemia cases, 506 were 


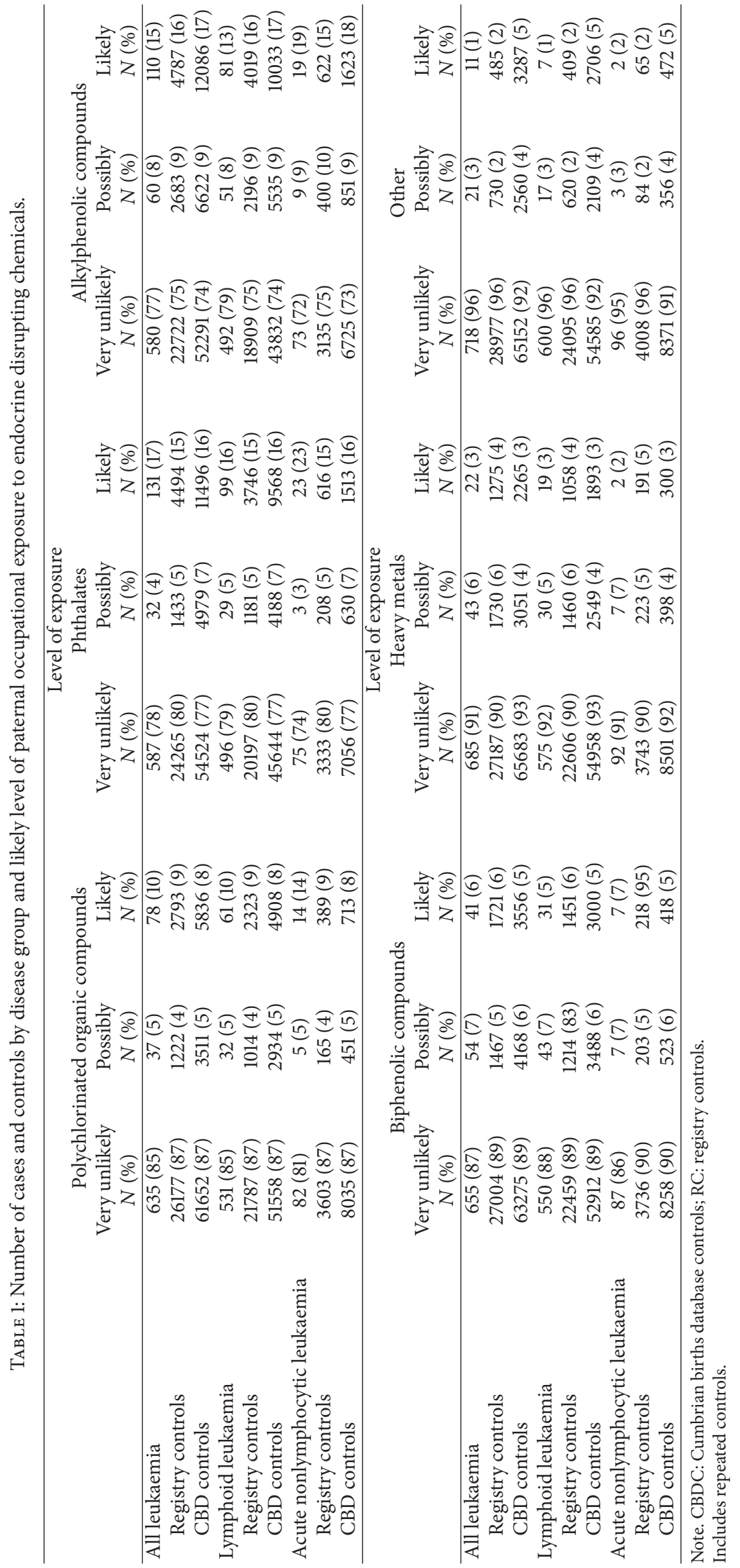


diagnosed before age 7 years (435 for lymphoid leukaemia and 59 for ANLL). Table 2 details the results of the conditional logistic regression analyses for each exposure group, for all leukaemia, lymphoid leukaemia, and ANLL, and by control group.

3.1. Polychlorinated Organic Compounds. There were significantly raised risks, using both sets of controls, of ANLL in the female offspring of fathers likely to have been exposed to polychlorinated organic compounds (unadjusted $P$ values $<0.001$ for CBD and 0.027 for registry controls, Table 2). On adjustment for paternal social class, the result using CBD controls maintained a high level of significance (OR 2.72, 95\% CI 1.29-5.75, $P=0.009$ ) but lost significance when using registry controls (OR 1.79, 95\% CI 0.84-3.82, $P=0.13$ ). Significantly raised risks of all leukaemias and ANLL were also seen when males and females were combined but were only significant when using CBD controls, with nonsignificantly raised odds ratios when using registry controls. Risk of lymphoid leukaemia in male offspring was also raised significantly when using registry controls $(P=0.039)$ but was only of borderline significance when using $\mathrm{CBD}$ controls $(P=0.077)$.

3.2. Phthalates. When using registry controls, there were significant associations between paternal likely phthalate exposure and risk of ANLL in both all offspring and all leukaemia in males. However, no significant associations were seen when using CBD controls.

3.3. Alkylphenolic Compounds. There was a significant association between paternal likely exposure to alkylphenolic compounds and reduced risk of lymphoid leukaemia. This was consistent between the cohorts $(P=0.006$ when using CBD controls and $P=0.021$ when using registry controls) but was restricted to female offspring in sex-specific analyses ( $P=0.003$ when using either set of controls). These inverse associations remained after adjustment for paternal occupational social class.

3.4. Biphenolic Compounds. The only significant association with paternal likely exposure to biphenolic compounds was with ANLL in female offspring when using CBD controls $(P=0.003)$. The magnitude and significance of this result reduced after adjustment for paternal occupational social class (OR=2.38, 95\% CI 0.89-6.39, $P=0.085)$. No significant association was seen when using registry controls.

3.5. Heavy Metals and "Other" Potential Endocrine Disrupting Chemicals. No significant associations were seen between paternal likely exposure to heavy metals and risk of leukaemia in offspring. Significant inverse associations were seen with likely exposure to "other" potential endocrine disrupting chemicals and risk of leukaemia, particularly lymphoid leukaemia. However, these associations were restricted to the analyses using $\mathrm{CBD}$ controls.

\section{Discussion}

Previous research has shown that endocrine disruptors can affect male and female reproduction, breast development and cancer, prostate cancer, neuroendocrinology, thyroid, metabolism and obesity, cardiovascular endocrinology, and childhood development [1,2]. In this large case-control study, we identified significantly increased risks of ANLL with likely paternal exposure, around the time of birth, to polychlorinated organic compounds and phthalates. A number of inverse or nonsignificant findings are also reported and other significant associations were limited to one control group only.

There was a significantly increased risk of leukaemia in offspring of fathers likely to have been exposed to polychlorinated organic compounds. Occupations likely to be exposed to these chemicals include those working with electrical equipment. A similar association was also seen by Ali et al. who found an increased risk of leukaemia in the offspring of men who worked in paper production or as electronic equipment assemblers [9], although this may also reflect our previously reported association between paternal exposure to EMF and risk of leukaemia in offspring [23]. However, Keegan et al. found no association between leukaemia risk and paternal occupation in paper production [18].

Phthalates are mainly found in plastics, paints, glues, and in the cosmetic industry [10]. We found significant associations between likely paternal phthalate exposure and risk of leukaemia in offspring when using registry controls, but not when using CBD controls. This inconsistency is reflected in previous research with some studies finding a significant increased risk with paternal exposure to paints [5-7], and others finding no significant associations with paints or plastics [12-14, 16-18]. Lowengart et al. found a significant association between paternal paint exposure and increased risk of childhood leukaemia, although this became insignificant when adjusted for concurrent solvent exposure [15].

Many occupations include risk of exposure to akylphenolic compounds including those working with plastics, metals, paints, textiles, wood dust, and coal [8]. They are also found in pesticides as a surfactant [10]. We found a significantly reduced risk of childhood leukaemia in those whose fathers were likely to be exposed to alkylphenolic compounds. This is the only known study to have found this association. Others looking at occupational groups within this exposure category have found an increased risk of leukaemia [5-9, 12, 24] or no association at all [12-18], with some studies showing different findings for different subgroups of this exposure category [12]. Therefore, our finding may be spurious.

Biphenolic compounds are used in the production of plastics and resins. Many of these resins are used to coat metal products and some are used in dentistry [10]. There were no significant associations found between likely paternal biphenolic compounds exposures and childhood leukaemia risk in this study or in other studies [14-18]. Similarly, we found no significant associations between likely paternal exposure to heavy metals and risk of leukaemia in offspring. Buckley et al. also found no association [16], but Keegan 


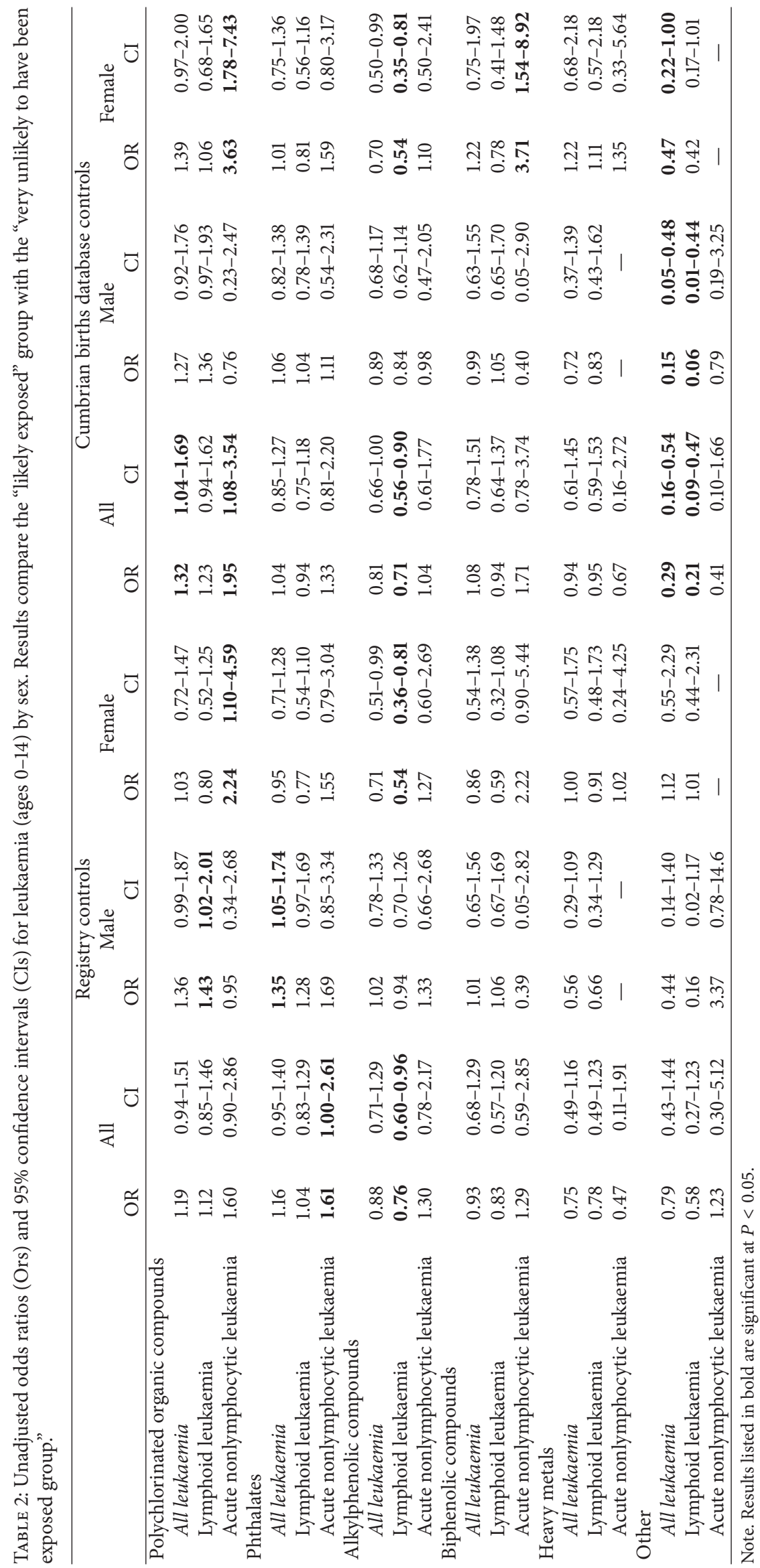


et al. found a protective association with paternal exposure to lead [18]. We found a significant inverse association between paternal likely exposure to "other" endocrine disrupting chemicals and the risk of childhood leukaemia. There is very little previous research in this category available for comparison, so this finding would require replication in another population to be interpretable.

It is not clear if paternal exposure to endocrine disruptors could have direct preconceptional carcinogenic effects. There is however evidence to suggest that exposure to endocrine disrupting chemicals may affect future generations through germline and nongenomic effects, the latter likely to include impacts on DNA methylation and histone acetylation [25]. Few studies of potential transgenerational effects consider the issue of sex, other than in terms of adjustment within a statistical model. Our finding of an association between childhood ANLL and likely paternal occupational exposure to polychlorinated organic compounds was restricted to female offspring. Reasons for this may include a lack of statistical power for male offspring, especially if the effect size is smaller or that it is a chance finding. However, other areas of epidemiological research, particularly those looking at the early origins of obesity or cardiovascular disease, suggest sexspecific transgenerational influences [26], suggesting that the potential for sex-specific associations with paternal exposures needs further consideration. Paternal occupation at birth was used, as this is the most likely source of paternal occupation data at a population level. While this should reflect likely exposure around the time of birth, it may also reflect a paternal preconceptional exposure, or a "carry-home" effect in which the father passes on the exposure (e.g., via exposed clothing, hair, or skin). This would particularly be relevant for the leukaemia cases in this study who were among the oldest at diagnosis.

Previous case-control studies investigating this topic have been limited by their small sample sizes. This study's major strengths are its size and population base. Over 700 cases of leukaemia were included from the NYPMDR, with good case ascertainment [19]. Bias in control selection was minimised by using routine data for both control groups. Matching on year of birth removed the likely confounding effect of time period. Occupations were taken from birth certificates eliminating recall bias. Findings were broadly similar using the two control groups, albeit with some important differences in statistical significance, suggesting that, where consistent, the observed results were unlikely to arise as the result of bias in control selection. Where inconsistent, it is possible that the findings are due to chance. This study also has some limitations, many of which apply to most of the studies in this field; the use of job title as a surrogate for exposure, lack of data on levels or timings of exposure, possible changes in exposure within occupational groups over the long study period, and multiple testing could potentially give rise to spurious results. It is also possible that the exposures included in this analysis could be correlated with other exposures already shown to increase cancer risk in offspring, such as solvents. There is also the possibility that other factors unavailable to this study, such as maternal occupational exposures or environmental or lifestyle factors in childhood or in early adult life, could explain the significant findings.

In conclusion, we have found evidence that likely paternal exposure to potential endocrine disruptors may be linked to an increased risk of leukaemia in offspring. However, the significant associations found in this study should be viewed with caution, especially those for which inconsistent results were seen between the control groups used. Further research in this area, perhaps with narrower and more accurate exposure categories, incorporating other potential confounding exposures to the mother and child, is needed before any firm conclusions can be drawn.

\section{Conflict of Interests}

The authors declare that there is no conflict of interests regarding the publication of this paper.

\section{Acknowledgments}

The NRYPMDR is funded by the Newcastle upon Tyne Hospitals NHS Foundation Trust, with research support from the North of England Children's Cancer Research Fund. The authors are grateful to Richard Hardy for computing support and to Katharine Kirton and Dr Jane Salotti for assistance with the Cumbrian births database.

\section{References}

[1] E. Diamanti-Kandarakis, J. P. Bourguignon, L. C. Giudice et al., "Endocrine-disrupting chemicals: an Endocrine Society scientific statement," Endocrine Reviews, vol. 30, no. 4, pp. 293342, 2009.

[2] J. D. Meeker, "Exposure to environmental endocrine disruptors and child development," Archives of Pediatrics and Adolescent Medicine, vol. 166, no. 10, pp. 952-958, 2012.

[3] J. Toppari, J. C. Larsen, P. Christiansen et al., "Male reproductive health and environmental xenoestrogens," Environmental Health Perspectives, vol. 104, no. 4, pp. 741-803, 1996.

[4] J. Little, Epidemiology of Childhood Cancer, vol. 149 of IARC Scientific, International Agency for Research on Cancer, Lyon, France, 1999.

[5] K. Hemminki, I. Saloniemi, T. Salonen, T. Partanen, and H. Vainio, "Childhood cancer and parental occupation in Finland," Journal of Epidemiology and Community Health, vol. 35, no. 1, pp. 11-15, 1981.

[6] P. A. McKinney, F. E. Alexander, R. A. Cartwright, and L. Parker, "Parental occupations of children with leukaemia in west Cumbria, North Humberside, and Gateshead," British Medical Journal, vol. 302, no. 6778, pp. 681-687, 1991.

[7] J. S. Colt and A. Blair, "Parental occupational exposures and risk of childhood cancer," Environmental Health Perspectives, vol. 106, no. 3, pp. 909-925, 1998.

[8] M. Vrijheid, B. Armstrong, H. Dolk, M. van Tongeren, and B. Botting, "Risk of hypospadias in relation to maternal occupational exposure to potential endocrine disrupting chemicals," Occupational and Environmental Medicine, vol. 60, no. 8, pp. 543-550, 2003.

[9] R. Ali, C. Yu, M. Wu et al., "A case-control study of parental occupation, leukemia, and brain tumors in an industrial city in 
Taiwan," Journal of Occupational and Environmental Medicine, vol. 46, no. 9, pp. 985-992, 2004.

[10] M. van Tongeren, M. J. Nieuwenhuijsen, K. Gardiner et al., "A job-exposure matrix for potential endocrine-disrupting chemicals developed for a study into the association between maternal occupational exposure and hypospadias," Annals of Occupational Hygiene, vol. 46, no. 5, pp. 465-477, 2002.

[11] M. S. Pearce, D. M. Hammal, M. T. Dorak, R. J. Q. McNally, and L. Parker, "Paternal occupational exposure to pesticides or herbicides as risk factors for cancer in children and young adults: a case-control study from the North of England," Archives of Environmental and Occupational Health, vol. 61, no. 3, pp. 138-144, 2006.

[12] M. Feychting, N. Plato, G. Nise, and A. Ahlbom, "Paternal occupational exposures and childhood cancer," Environmental Health Perspectives, vol. 109, no. 2, pp. 193-196, 2001.

[13] S. L. Kwa and L. J. Fine, "The association between parental occupation and childhood malignancy," Journal of Occupational Medicine, vol. 22, no. 12, pp. 792-794, 1980.

[14] H. A. van Steensel-Moll, H. A. Valkenburg, and G. E. van Zanen, "Childhood leukemia and parental occupation. A registerbased case-control study," The American Journal of Epidemiology, vol. 121, no. 2, pp. 216-224, 1985.

[15] R. A. Lowengart, J. M. Peters, C. Cicioni et al., "Childhood leukemia and parents' occupational and home exposures," Journal of the National Cancer Institute, vol. 79, no. 1, pp. 3946, 1987.

[16] J. D. Buckley, L. L. Robison, R. Swotinsky et al., "Occupational exposures of parents of children with acute nonlymphocytic leukemia: a report from the Childrens Cancer Study Group," Cancer Research, vol. 49, no. 14, pp. 4030-4037, 1989.

[17] J. Schuz, U. Kaletsch, R. Meinert, P. Kaatsch, and J. Michaelis, "Risk of childhood leukemia and parental self-reported occupational exposure to chemicals, dusts, and fumes: results from pooled analyses of German population-based case-control studies," Cancer Epidemiology Biomarkers and Prevention, vol. 9, no. 8, pp. 835-838, 2000.

[18] T. J. Keegan, K. J. Bunch, T. J. Vincent et al., "Case-control study of paternal occupation and childhood leukaemia in Great Britain, 1962-2006," British Journal of Cancer, vol. 107, no. 9, pp. 1652-1659, 2012.

[19] S. J. Cotterill, L. Parker, A. J. Malcolm, M. Reid, L. More, and A. W. Craft, "Incidence and survival for cancer in children and young adults in the North of England, 1968-1995: a report from the Northern Region Young Persons' Malignant Disease Registry," British Journal of Cancer, vol. 83, no. 3, pp. 397-403, 2000.

[20] L. Parker, J. Smith, H. Dickinson et al., "The creation of a database of children of workers at a nuclear facility: an exercise in record linkage," Applied Occupational and Environmental Hygiene, vol. 12, no. 1, pp. 40-45, 1997.

[21] M. S. Pearce, L. Parker, K. P. Windebank, S. J. Cotterill, and A. W. Craft, "Cancer in adolescents and young adults aged 1524 years: a report from the North of England Young Person's Malignant Disease Registry, UK," Pediatric Blood and Cancer, vol. 45, no. 5, pp. 687-693, 2005.

[22] Office of Population Census and Surveys, Standard Occupational Classification Volumes 1,2,3, Her Majesty's Stationery Office, London, UK, 1990.

[23] M. S. Pearce, D. M. Hammal, M. T. Dorak, R. J. Q. McNally, and L. Parker, "Paternal occupational exposure to electro-magnetic fields as a risk factor for cancer in children and young adults: a case-control study from the North of England," Pediatric Blood \& Cancer, vol. 49, no. 3, pp. 280-286, 2007.

[24] C. Magnani, G. Pastore, L. Luzzatto, and B. Terracini, "Parental occupation and other environmental factors in the etiology of leukemias and non-Hodgkin's lymphomas in childhood: a casecontrol study," Tumori, vol. 76, no. 5, pp. 413-419, 1990.

[25] M. D. Anway and M. K. Skinner, "Epigenetic transgenerational actions of endocrine disruptors," Endocrinology, vol. 147, no. 6, pp. S43-S49, 2006.

[26] M. E. Pembrey, L. O. Bygren, G. Kaati et al., "Sex-specific, maleline transgenerational responses in humans," European Journal of Human Genetics, vol. 14, no. 2, pp. 159-166, 2006. 


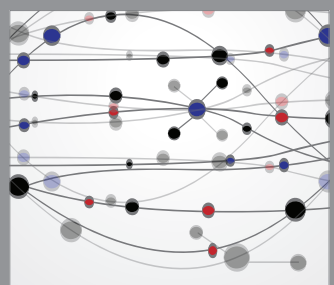

The Scientific World Journal
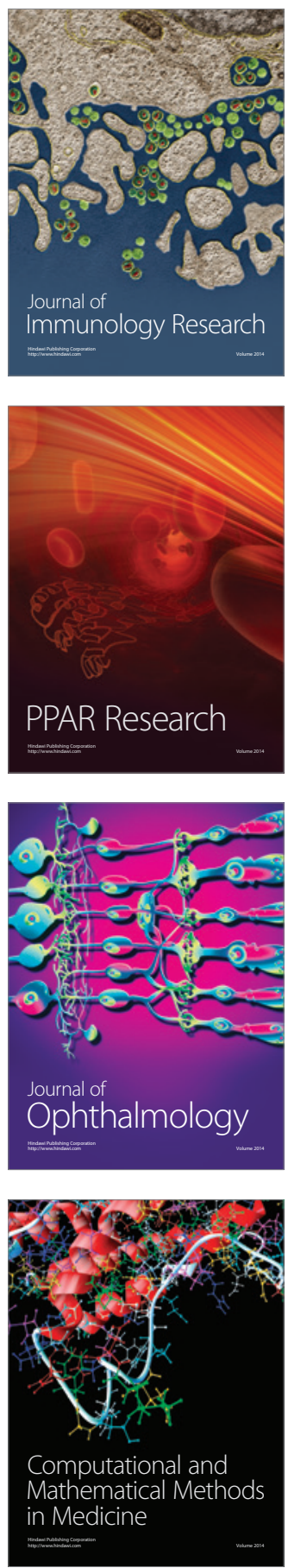

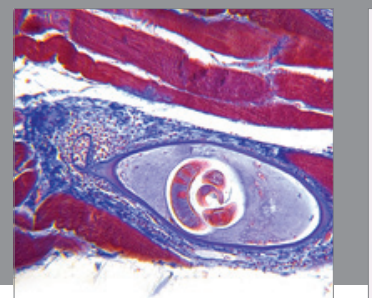

Gastroenterology

Research and Practice
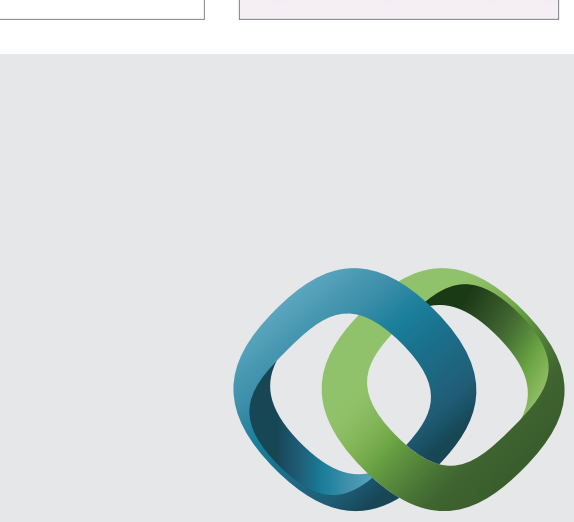

\section{Hindawi}

Submit your manuscripts at

http://www.hindawi.com
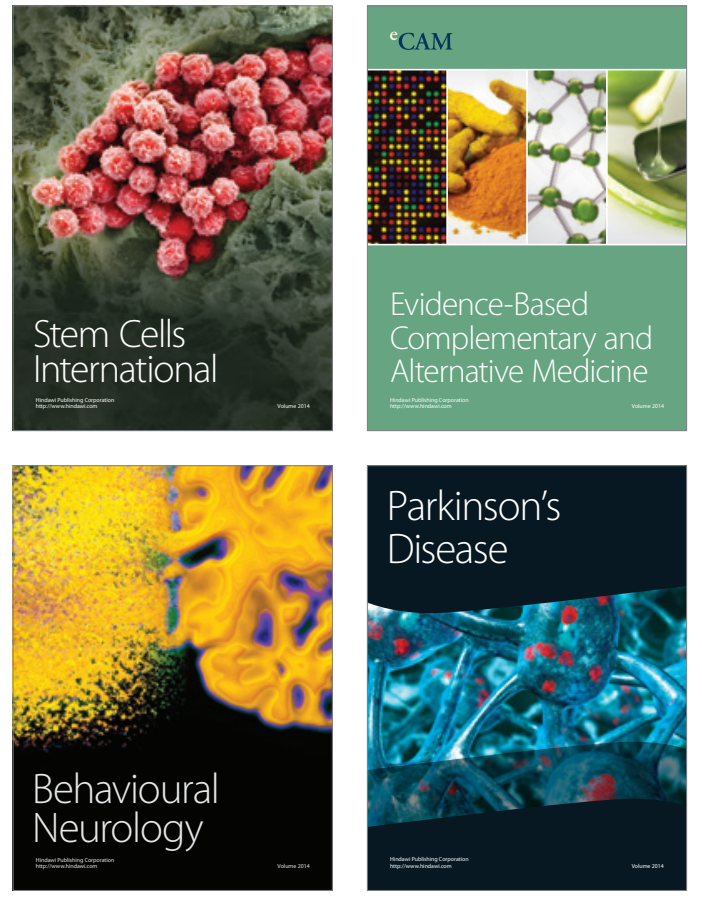
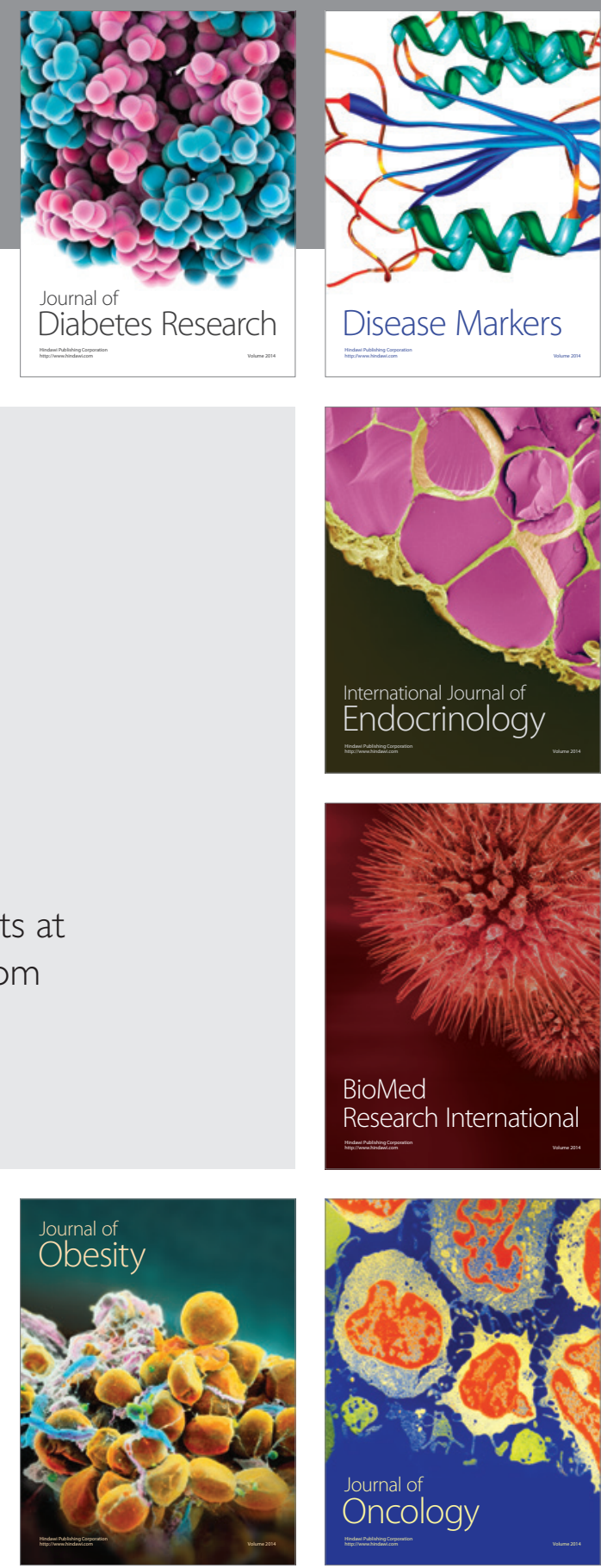

Disease Markers
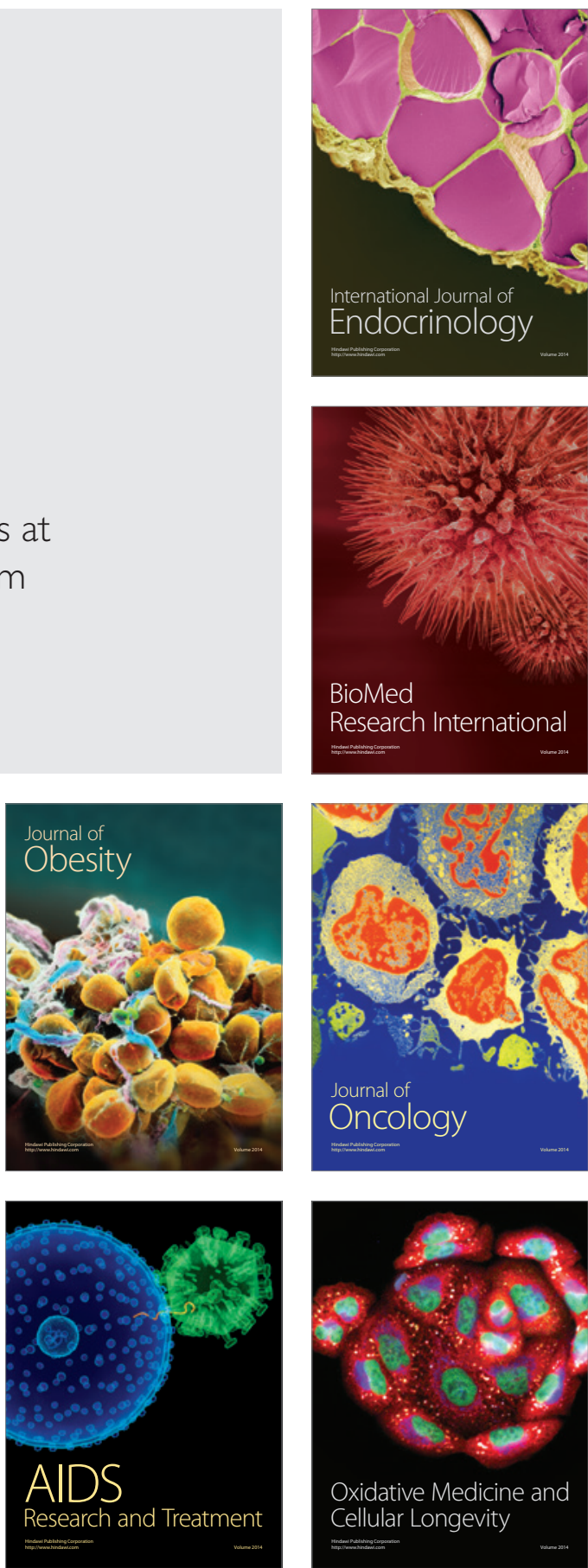